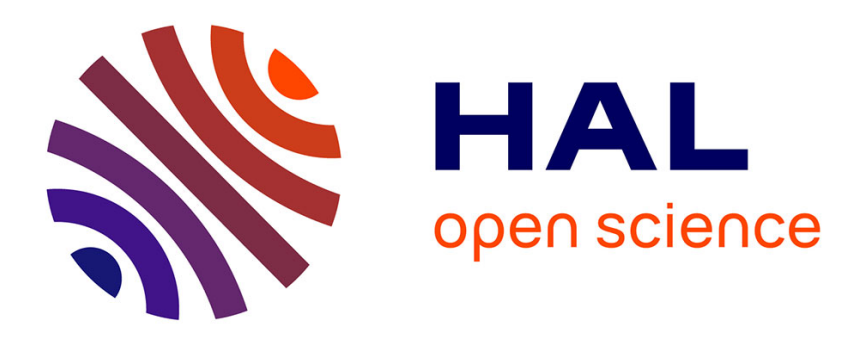

\title{
Dynamics and Topology of Micromagnetic Vortices
}

Alberto Verga

\section{To cite this version:}

Alberto Verga. Dynamics and Topology of Micromagnetic Vortices. Chaos, Complexity and Transport

CCT'15, Jun 2015, Marseille, France. pp.54 - 82, 10.1142/9789813202740_0002 . hal-01500461

\section{HAL Id: hal-01500461 \\ https://hal-amu.archives-ouvertes.fr/hal-01500461}

Submitted on 3 Apr 2017

HAL is a multi-disciplinary open access archive for the deposit and dissemination of scientific research documents, whether they are published or not. The documents may come from teaching and research institutions in France or abroad, or from public or private research centers.
L'archive ouverte pluridisciplinaire HAL, est destinée au dépôt et à la diffusion de documents scientifiques de niveau recherche, publiés ou non, émanant des établissements d'enseignement et de recherche français ou étrangers, des laboratoires publics ou privés. 


\title{
Dynamics and Topology of Micromagnetic Vortices
}

\author{
Alberto D. Verga* \\ Centre de Physique Théorique, Aix-Marseille University, \\ Campus de Luminy, 13288 Marseille cedex 09, France \\ *E-mail: alberto.verga@univ-amu.fr
}

\begin{abstract}
Magnetic vortices are topologically stable magnetization configurations at the nanoscale. When subject to a current polarized in spin, exchange interaction with the itinerant electrons, modify the configuration of the fixed spins. The spin-transfer torque effect, as experimentally observed, is able to change the magnetization topology. We investigate the relation between the dynamics of magnetic vortices driven by a spin-polarized current and the changes in the magnetization topology. These transitions are characterized by the appearance of magnetic singularities.
\end{abstract}

Keywords: Magnetism; Magnetic Vortices; Landau-Lifshitz equation; Spintransfer torque; Magnetic singularities.

\section{Introduction}

The elementary theory of magnetism was introduced in the early twenty century by Weiss, Curie and Bloch. They described the ferromagneticparamgnetic transition, the existence of magnetic domains, and the hysteresis of magnetic materials. When an external magnetic field $\mu_{0} H_{z}$ is applied to a ferromagnetic material, it acquires a net magnetization $\boldsymbol{M}$ in the direction of the applied field (Fig. 1). For relatively weak fields, the magnetization of soft materials (materials having a thin hysteresis cycle) saturates, and the value of saturation is no far from the zero field value. Under such conditions, at temperatures much smaller than the Curie temperature, one may consider that the entire body magnetization is at its saturation value. This approximation defines the validity of micromagnetism, an approximation particularly well suited to the microscopic scales. The magnetization can then be described by a vector in the unit sphere, the Bloch sphere.

Already in 1935 Landau and Lifshitz ${ }^{1}$ settled the bases of the micromagnetic theory. In particular, they introduced, before the general theory of phase transitions, the fundamental concepts of order parameter and of the 


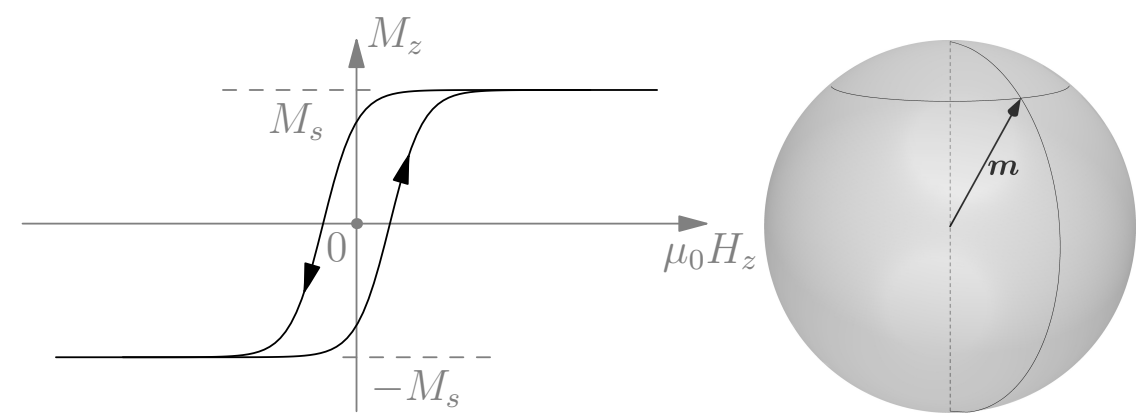

Fig. 1. Magnetization $\boldsymbol{M}$ as a function of an external magnetic field $H_{z}$ (left) and the unit magnetization vector $\boldsymbol{m}=\boldsymbol{M} /|\boldsymbol{M}|$ in the Bloch sphere (right). Below the Curie temperature, a ferromagnetic material is characterized by a saturated magnetization of contant magnitude $M_{s}$.

effective free energy functional of the order parameter. In this novel framework, thermodynamics of homogeneous and uniform systems can therefore be generalized to take into account spatial gradients, anisotropy and finite size effects. Landau and Lifshitz predicted that the magnetization of a ferromagnetic crystal cannot be uniform, instead it forms magnetic domains with a given magnetization orientation, separated by walls. In fact, a state characterized by a magnetization spatially uniform, is a statistical property of an infinite system valid, in principle, in the thermodynamic limit. However, for a finite volume the surface demagnetizing field (dipolar field) naturally determines the size of the constant magnetization domains and their geometry. The minimization of the free energy functional gives the stable states of the ferromagnet. The existence of a finite value of the magnetization below the Curie point, is explained by the behavior of the free energy that can be expanded in even powers of the magnetization near the transition: a sign change of the second order coefficient with the temperature gives the characteristic exponents of the thermodynamics quantities. In addition, a variational principle, equivalent to the principle of least action in mechanics, leads to the equations governing the dynamics of the order parameter. For instance, one can describe the motion of the walls separating domains with different magnetization, under the action of an external field.

In a remarkable paper, Belavin and Polyakov, ${ }^{2}$ asked whether an infinite magnetic film can support inhomogeneous states, at variance with the thermodynamic expectation of an uniform state. They found that, in the limit where the free energy is dominated by the exchange interaction, 
localized structures of finite energy are possible. These structures, already proposed by Skyrme ${ }^{3}$ in his theory of strong forces and now called after him "skyrmions," are endowed with a nontrivial topology: they cannot be deformed into an uniform field without introducing a singularity in the magnetization field (a point of zero magnetization). ${ }^{4}$ skyrmions were recently discovered in chiral metals and other materials having strong spin-orbit coupling energy. ${ }^{5,6}$

The goal of the present paper is to present and expand recent results in the study, beyond the limits of the micromagnetic theory, of localized magnetization structures, skyrmions in particular, under the action of a spin-polarized current. Our main interest is in the topological changes, which involve within the micromagnetic model, the appearance of magnetization singularities.

We start with the Landau and Lifshitz theory of micromagnetism, we present relevant experimental results showing magnetic vortices in nanoferromagnets ${ }^{7}$ and the methods to change the magnetic configurations using fields and currents. ${ }^{8}$ We then propose a model to generalize the micromagnetic framework in order to account for the atomic scales involved in the topological changes. ${ }^{9}$ Finally, we present in particular a quantitative analysis of the skyrmion transition to a ferromagnetic state. ${ }^{10}$

\section{Vortices and skyrmions}

When the dimensions of a ferromagnet shrink below micrometer scales, one observes that the shape and size of the magnetic domains change. ${ }^{11}$ Magnetic textures with a nontrivial topology spontaneously organize at nanometric scales. Sixteen years ago, Shinjo et al. ${ }^{7}$ found magnetic vortices imaging the magnetization distribution in circular dots of permalloy with a magnetic force microscope; typical dimensions of the ferromagnets were about $1 \mu \mathrm{m}$ in diameter and $50 \mathrm{~nm}$ thick. Magnetic vortices are essentially curly distributions of the magnetization with a central core out of the plane (Fig. 2). The in-plane curly distribution minimizes the dipolar field (field lines around the magnet) and the out-of-plane central region, the vortex core, minimizes the exchange energy of neighboring spins. The size of the core is only a few nanometers, and is determined by the competition between dipolar and exchange energies.

In addition to magnetic vortices, a physical realization of skyrmions were theoretically predicted by Bogdanov et al. ${ }^{12,13}$ in metals possessing a strong spin-orbit interaction of the type first described by Dzyaloshinskii 


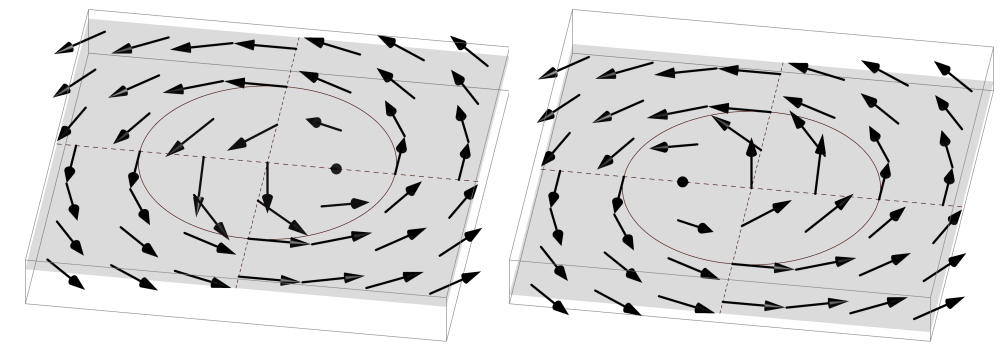

Fig. 2. Schematic magnetization distribution of a vortex of negative charge (left) and positive charge (right). The topological charge depends on the number of turns of the vector field around the origin, and the polarity of the core, up or down. The tangential direction of the field near the boundary, ensures the minimization of the dipolar field. The out of the plane magnetization near the center (vortex core) avoid large gradients, minimizing the exchange energy.

and Moriya. Isolated and periodic lattices of skyrmions were indeed discovered in chiral metals ${ }^{5,14}$ and ferrimagnets ${ }^{15}$. The Dzyaloshinskii-Moriya spin-orbit interaction tends to stabilize the skyrmion textures in such a way that a skyrmion lattice appears as a genuine thermodynamic phase in helical materials. It is interesting to note that these lattices can be manipulated by relatively weak magnetic fields, currents, ${ }^{16}$ and even electric fields, ${ }^{17}$ opening the way up to applications in the domain of spintronics and magnetic memories. ${ }^{18,19}$

One crucial property of magnetic textures in nanoferromagnets is that they possess a nontrivial topology: the magnetization field cannot be continuously deformed to obtain an uniform field (ferromagnetic state). This impossibility is related with the conservation of the magnetization magnitude, the vector $\boldsymbol{m}$ cannot vanish. Textures differing in their topology can be classified according to their topological charge

$$
Q=\int_{\Sigma} \frac{\mathrm{d} \boldsymbol{x}}{4 \pi} \boldsymbol{m} \cdot\left(\frac{\partial \boldsymbol{m}}{\partial x} \times \frac{\partial \boldsymbol{m}}{\partial y}\right)
$$

The topological charge of two dimensional configurations, has a simple geometrical interpretation. It represents the solid angle swept by the unit vector on the sphere when mapped from the plane to the Bloch sphere. A pair of vortices of opposite polarity cover the whole sphere, their combined charge is one; from this one deduces that each vortex is of charge one half.

In their experiments, van Waeyenberge et al. ${ }^{20}$ use permalloy square elements of about one micron side and $50 \mathrm{~nm}$ thick. A texture containing a central vortex is perturbed by a pulse of an in-plane magnetic field. 

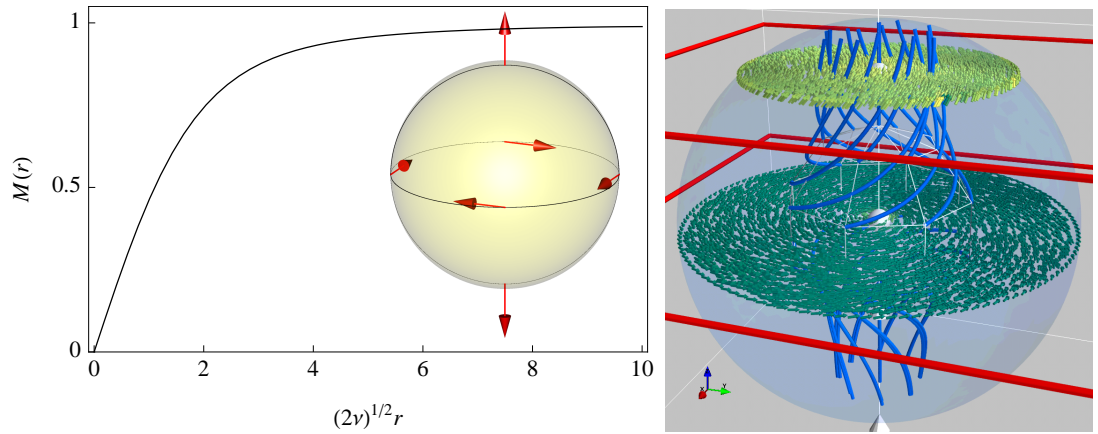

Fig. 3. Magnetization in the core region of a Bloch point. (Left) The magnitude of the magnetization vanishes at the center. (Right) Twisted lines of equal magnetization, showing the structure of a Bloch point. Adapted from Ref. $\left.{ }^{24}\right]$.

The field switch the core polarization of the vortex, from a core up (as in Fig. 2 right) to a core down (Fig. 2 left). The duration of the burst is $4 \mathrm{~ns}$ and its amplitude $1.5 \mathrm{mT}$; orders of magnitude smaller than the one necessary to switch the magnetization with a static field. The change in the vortex polarization, is a change in its topology, normally forbidden in the micromagnetic framework with $|\boldsymbol{M}|=$ const. Physical processes outside the scope of this model are necessarily at work: loss of regularity and dissipation can be at the origin of micromagnetic singularities necessarily present during the transition between distinct states.

As observed by Hertel and others, ${ }^{21-23}$ vortex switch involves an intermediate stage in the form of a Bloch point. A Bloch point is a three dimensional texture with a magnetization field covering all space directions: the interface between two magnetic domains is a domain wall; the intersection of two walls, is a singular line; the intersection of two Bloch lines is a Bloch point. A Bloch point is a peculiar magnetic structure, necessarily singular, because the magnetization must vanish somewhere inside its volume (Fig. 3). The variation of the topological charge (1) that accompanies the vortex switch is $\Delta Q=1 / 2-(-1 / 2)=1$, this variation precisely corresponds to the charge of a Bloch point $Q_{B}$; the whole process can be written as a balance equation of the topological charge:

$$
Q_{1}+Q_{B}=-1 / 2+1=1 / 2=Q_{2},
$$

where $Q_{1,2}$ are the vortex charges.

In order to change the topology of the magnetization field, an alternative to magnetic field perturbations, is to apply electric currents polar- 
ized in spin. One remarkable property of ferromagnetic materials is that in addition to support a wealth of stable static configurations, they are also metallic and able to carry a current. However, at variance to ordinary metals, a conduction electron in a ferromagnet tends to align its spin in the direction of the ambient magnetization, and then to form a spinpolarized current. The opposite of this mechanism, the transfer of angular momentum carried by the current to the magnetic texture, modifying its configuration, is called spin-transfer torque. This effect was theoretically predicted by Slonczeswki and Berger, using different approaches and model systems, in 1996. ${ }^{25,26}$ Under controlled experimental conditions one can design ferromagnetic heterostructures containing for instance a free spin layer in contact, through a tunnel barrier (a thin layer of normal metal), with a fixed magnetization ferromagnet used to polarize an electric current. Various experiments based on this setup, confirmed the basic predictions of the theoretical models advanced by Berger and Slonczewski. ${ }^{27,28}$ Spintransfert torque is also responsible of the motion of a domain wall pushed by pulses of a polarized current. ${ }^{29}$

A striking and technologically interesting application of the spin-transfer torque effect was revealed by Rommer et al. ${ }^{8}$ some years ago. They demonstrated that applying a spin-polarized current with a scanning tunneling microscope on a thin film consisting in a palladium-iron bilayer, it is possible to "write" and to "delete" skyrmions in a controlled way. This is an example of nucleation of skyrmions by spin-transfer torque effect, and of the transition between this topological skyrmion state and a trivial one, the homogeneous ferromagnetic state:

$$
\text { skyrmion } \rightarrow \Delta Q=1 \rightarrow \text { ferromagnetic . }
$$

Therefore, experiments show that a spin-polarized current induces topological phase changes, like the skyrmion-ferromagnetic transition.

\section{Micromagnetic equations}

In the preceding section various physical mechanisms were identified as being important to describe magnetic textures in microscopic magnets:

- The exchange interaction, proportional to the magnetization gradients, characterized by the "exchange stiffness" constant $A$, having the dimensions of an energy per length unit; it defines the exchange length $\ell=\sqrt{A / \mu_{0} M_{s}^{2}}$, which gives an order of magnitude of the 
vortex cores size. It of the order of a few nanometers for common transition metals.

- The magnetic anisotropy, that can be related to the crystal structure, or an effect of the geometry and in this case a form of the dipolar energy, may determine an "easy plane" or an "easy axis". For permalloy discs (magnetic dots), the magnetization is confined on the disk plane (easy plane); for magnetic films, often the magnetization is out of the plane (easy axis), which is positive for the easy plane case and negative for the easy axis case. The simplest model of magnetic anisotropy includes only one constant $K$ (energy per unit volume). A anisotropy characteristic length $\ell_{K}=\left(\mu_{0} M_{s}^{2} a^{3} / K\right)$, where $a$ is of the order of the lattice step, can be as small as $a$.

- The spin-orbit interaction that leads to the Dzyaloshinkii-Moryia magnetic energy contribution, favoring the helical alignement of spins. This interaction is proportional to the rotational of the magnetization field, through a coupling constant $D$ whose sign determines the chirality of the helical textures.

- The long range dipolar interactions created by the volume and surface magnetization charges: the divergence of the magnetization is the source of a "stray field" that couples with the body's magnetization itself. This interaction is essential in establishing the whole magnetization distribution in accordance with the geometry of the ferromagnet, but can be neglected when considering the dynamics of the vortex cores, dominated by the large magnetization gradients and therefore, by the exchange energy.

- The interaction with a stream of spin polarized electrons, giving rise to a transfer of angular momentum between itinerant and fixed spins; the phenomenological description of the spin-transfer torque involves a current density $\boldsymbol{j}_{s}=n_{0} \boldsymbol{v}_{s}$, where the effective velocity $v_{s}$ is proportional to the current polarization $P=\left(n_{\uparrow}+n_{\downarrow}\right) /\left(n_{\uparrow}+n_{\downarrow}\right)$ ( $n_{0}=n_{\uparrow}+n_{\downarrow}$ is the electron density), coupled with a gauge vector potential $\boldsymbol{A}:{ }^{30,31}$

$$
\delta A_{k}=-\frac{\hbar}{2} \delta \boldsymbol{m} \cdot\left(\boldsymbol{m} \times \frac{\partial}{\partial x_{k}} \boldsymbol{m}\right) .
$$

The presence of $\hbar$ reveals the quantum origin of the spin-transfer torque, a point we discuss later.

In summary, a magnetic material can be described by an order param- 


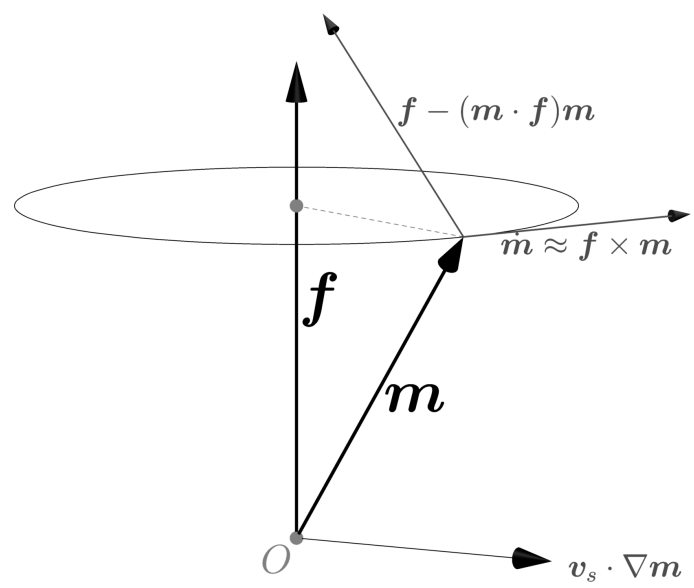

Fig. 4. Schematic representation of the magnetization $\boldsymbol{m}$ vector precessing around the self-consistent effective field $\boldsymbol{f}$, and spiraling towards $\boldsymbol{f}$ due to the $\alpha$ dissipation (direction $\boldsymbol{f}-(\boldsymbol{m} \cdot \boldsymbol{f}) \boldsymbol{m})$. We also show the motion, driven by the current $\boldsymbol{v}_{s}$, of the origin in the direction of the magnetization gradient $\boldsymbol{v}_{s} \cdot \nabla \boldsymbol{m}$, projected on the direction of the polarized current.

eter, the magnetization, and a Landau free energy functional

$$
F[\boldsymbol{M}]=\int_{\mathrm{vol}} \mathrm{d} \boldsymbol{x}\left[A(\nabla \boldsymbol{M})^{2}+K M_{z}^{2}-D \boldsymbol{M} \cdot \nabla \times \boldsymbol{M}-\mu \boldsymbol{M} \cdot \boldsymbol{H}_{d}\right]
$$

that takes into account the different microscopic interactions: exchange, anisotropy, spin-orbit, dipolar, and the additional term of coupling with itinerant electrons:

$$
F_{s}=\int_{\mathrm{Vol}} \mathrm{d} \boldsymbol{x} \boldsymbol{j}_{s} \cdot \boldsymbol{A}[\boldsymbol{m}]
$$

where $\boldsymbol{A}$ is the effective vector potential (2) associated with the accumulated Berry phase of a moving electron in a canted magnetization field. ${ }^{32}$ Variation of the free energy functional $F+F_{s}$ leads to the Landau-Lifshitz equation:

$$
\left(\frac{\partial}{\partial t}+\boldsymbol{v}_{s} \cdot \nabla\right) \boldsymbol{m}+\boldsymbol{m} \times \boldsymbol{f}=\alpha \boldsymbol{m} \times\left(\frac{\partial}{\partial t}+\frac{\beta}{\alpha} \boldsymbol{v}_{s} \cdot \nabla\right) \boldsymbol{m}
$$

generalized to account for the spin-transfer torque induced by a spin polarized current. The effective field $\boldsymbol{f}$ is defined by,

$$
\boldsymbol{f}=-\frac{\gamma}{M_{s}} \frac{\delta F}{\delta \boldsymbol{m}}
$$

has frequency units ( $\gamma$ is the gyromagnetic ratio). 
In addition to these conservative terms, we introduced on the right hand side of (5), two dissipation terms, which nevertheless preserve the magnetization magnitude $|\boldsymbol{M}|=M_{s}=$ const.:

- The first term, proportional to the nondimensional parameter $\alpha$, already present in the original Landau-Lifshitz treatment, tends to align the precessing magnetization in the direction of the selfconsistent field $\boldsymbol{f}$, it is sometimes referred as the Gilbert term.

- The second one, in $\beta$, is essentially a phenomenological term that breaks the Galilean invariance of the magnetization dynamics, and is related to the so-called non-adiabatic torque; it is a consequence of the dissipative loss, due to inelastic scattering of the electron's angular momentum. ${ }^{33}$ It appears as an effective magnetic field proportional to the gradient of the magnetization, and as a consequence, can control the asymptotic motion of a domain wall or other strongly inhomogeneous magnetization textures.

See Fig. 4 for a schematic representation of the different contributions to the magnetization evolution.

In order to explain the topological changes, involving the formation of singular structures as Bloch points, we must go beyond the framework of micromagnetism, and take into account processes possibly leading to loss of regularity.

\section{Semi-classical spin-transfer torque}

Magnetism is a quantum phenomenon. Although the micromagnetic model, based on the Landau free energy functional, efficiently describes the magnetization dynamics of ferromagnets up to the nanoscale, it is unable to follow the transition between distinct topological phases. ${ }^{4,9,34,35}$ The validity of the micromagnetic approach is limited by the formation of singularities, as the mentioned Bloch point, which reveals the existence of relevant scales at the atomic level, where quantum phenomena becomes unavoidable.

Our objective is to generalize the Landau-Lifshitz micromagnetic framework in order to introduce physical mechanisms with the aim to explain the change of topology induced by an electron current. As these changes involve sub-nanometric scales, we propose a model where the itinerant spins are treated quantum mechanically; in addition, the fixed spins, although treated classically, are supposed to be distributed in a lattice. The free energy of the fixed spins tends to the free energy functional of the magne- 
tization in the continuous limit. The coupling between itinerant and fixed spin is simply given by an exchange interaction.

With respect to the standard micromagnetic model (5), the semiclassical model differs in

- the treatment of the spin-transfer torque, terms in $\boldsymbol{v}_{s}$ are replaced by a coupling term with itinerant spins obeying Schrödinger equation; ${ }^{9}$ and

- the treatment of dissipation, the $\beta$ term is suppressed, and eventually a new exchange energy dissipation effect is considered. ${ }^{36}$

For instance, macroscopic magnetization arises from the microscopic exchange interaction between neighboring localized spins, as first described by the Heisenberg Hamiltonian on a crystal lattice,

$$
H_{\mathrm{xc}}=-J \sum_{\langle i, j\rangle} \boldsymbol{S}_{i} \cdot \boldsymbol{S}_{j}
$$

where $\boldsymbol{S}_{i}$ is a fixed spin at node $i$, coupled to a neighbor at site $j$ with exchange energy $J$. In addition to the localized spins, ferromagnetic materials also have delocalized conduction electrons that naturally polarize in the direction of the ambient magnetization. In the celebrated Stoner model one distinguish between minority and majority electronic bands, and the difference in their population gives the polarization parameter $P$. One consequence is that scattering processes become sensitive to the spin direction, leading to a spin dependent electric transport. For instance, the magnetic resistance $R \sim 1 /(1-P)$, may diverge for layers with $P=100 \%$ polarization. The opposite effect, the modification of the magnetization by the spin of the conduction carriers scattered by magnetization gradients, is the spin-transfer torque.

In addition to the lattice spins, there are also the conduction spins whose coupling with the fixed ones give rise to the spin-transfer torque. This interaction with the itinerant electrons can be taken into account through an exchange constant $J_{s}$ between $d$ and $s$ orbitals, corresponding respectively to the fixed (magnetization) spins and the conduction (current carriers) spins,

$$
H_{\mathrm{sd}}=J_{s} \sum_{i} \boldsymbol{S}_{i} \cdot \hat{\boldsymbol{s}}_{i},
$$

where $\hat{\boldsymbol{s}}_{i}$ is the electron spin operator:

$$
\hat{\boldsymbol{s}}_{i}=\frac{\hbar}{2} c_{i}^{\dagger} \boldsymbol{\sigma} c_{i}, \quad c_{i}=\left(\begin{array}{c}
c_{\uparrow i} \\
c_{\downarrow i}
\end{array}\right)
$$


$\boldsymbol{\sigma}$ is the vector of Pauli matrices, and $c_{i}$ the annihilation operator of an electron at site $i$. The interaction Hamiltonian contains the s-d coupling of itinerant and fixed spins $J_{s}$, which is in general larger than the Heisenberg exchange constant $J$, and determines the atomic time scale, which is of the order of the femtosecond.

It is worth noting that the Heisenberg Hamiltonian (7) leads to the exchange term of the Landau free energy, in the continuum limit: $a \rightarrow 0$, the lattice size tending to zero, and $N \rightarrow \infty$, the number of sites tending to infinity, with

$$
A=J / 2 a, \quad \boldsymbol{M}=-\frac{\hbar \gamma}{a^{3}}\langle\boldsymbol{S}(\boldsymbol{x})\rangle
$$

where $\gamma$ is the gyromagneitc factor. Therefore, we can generalize the micromagnetic model to atomistic scales, by a systematic discretization of the free energy terms. The advantage of this approach is that the conduction electrons can be treated quantum mechanically. We restrict our model to two dimensions, and neglect the long range interactions. These approximations are justified, if the goal is to describe the basic mechanisms at work in the formation of the singularities, where exchange interactions are naturally dominant. Two dimensions are enough to investigate the skyrmion-ferromagnetic transition, because the process do not involve the formation of a Bloch point (a three dimensional object), as in the switching or annihilation of magnetic vortices.

An appropriated semiclassical model to describe the physics of the topological transitions including atomic scales effects, is defined by a lattice of fixed classical spins interacting via the s-d Hamiltonian with quantum hopping electrons. Taking fixed spins as classical is in accordance with its slow and smooth dynamics compared with the moving electrons. In order to match the quantum dynamics of the electrons with the magnetization dynamics, some averaging process must be invoked. As a consequence, the itinerant spins appear at the scales of the fixed ones, as a stochastic perturbation, as we discuss later. It is this stochasticity of the whole system's dynamics that allows ultimately to investigate the changes in the topology of the magnetic textures.

In its most general form, we assume that fixed spins are coupled by a Heisenberg exchange Hamiltonian $H_{\mathrm{xc}}(7)$, and that the crystal structure gives rise to an easy-plane or easy-axis anisotropy $H_{\text {ani }}$ according to the sign of $K$, and to spin-orbit coupling $H_{\mathrm{DM}}$ that supports helical order in ferromagnetic films. Collecting these contributions we get the fixed spin 
Hamiltonian

$$
H_{S}=H_{\mathrm{xc}}+H_{\mathrm{ani}}+H_{\mathrm{DM}}
$$

with

$$
\begin{aligned}
H_{\mathrm{xc}} & =-\frac{J}{2} \sum_{i}\left(\nabla \boldsymbol{S}_{i}\right)^{2} \\
H_{\mathrm{ani}} & =\frac{K}{2} \sum_{i}\left(\boldsymbol{S}_{i} \cdot \hat{\boldsymbol{z}}\right)^{2} \\
H_{\mathrm{DM}} & =-\frac{D}{2} \sum_{i} \boldsymbol{S}_{i} \cdot \nabla \times \boldsymbol{S}_{i}
\end{aligned}
$$

where $\nabla$ is the discrete gradient and $\hat{\boldsymbol{z}}$ is the direction perpendicular to the lattice plane; the new parameters $K$ and $D$, are characteristic energies related to the phenomenological parameters of the Landau-Lifshitz equation (they differ in dimensional factors, in powers of the lattice constant $a$ ).

Fixed spins are coupled with conduction electrons by the s-d Hamiltonian (8). We consider that these electrons are subject to a difference of electric potential that creates a current, and to a crystal effective magnetic field that tends to polarize their spins. Therefore, the whole system is open, under the influence of an external electric field and a polarization magnetic field acting on free electrons. In order to preserve translational invariance we gauge out the electric field, introducing an appropriated time dependent quantum phase. The electron Hamiltonian that takes into account these effects is then,

$$
H_{e}=-\epsilon \sum_{\langle i, j\rangle} \mathrm{e}^{\mathrm{i} \phi_{i, j}(t)} c_{i}^{\dagger} c_{j}-\boldsymbol{B}_{p} \cdot \sum_{i} c_{i}^{\dagger} \boldsymbol{\sigma} c_{i},
$$

where the first term is the hopping energy between neighboring lattice sites $i, j$, the second term is the polarization field $B_{p}$, and the electric phase (gauge vector potential) is

$$
\phi_{i, j}(t)=(i-j) \cdot \hat{x} \frac{e a E t}{\hbar}
$$

here $a$ is the lattice constant, and $\epsilon$ the hopping energy. The spin of the electron is given by the quantum mean,

$$
\boldsymbol{s}_{i}(t)=\left\langle 0\left|c_{i}^{\dagger}(t) \boldsymbol{\sigma} c_{i}(t)\right| 0\right\rangle
$$

in the state with wave function,

$$
\psi\left(\boldsymbol{x}_{i}, t\right)=\left\langle\boldsymbol{x}\left|c_{i}^{\dagger}(t)\right| 0\right\rangle
$$



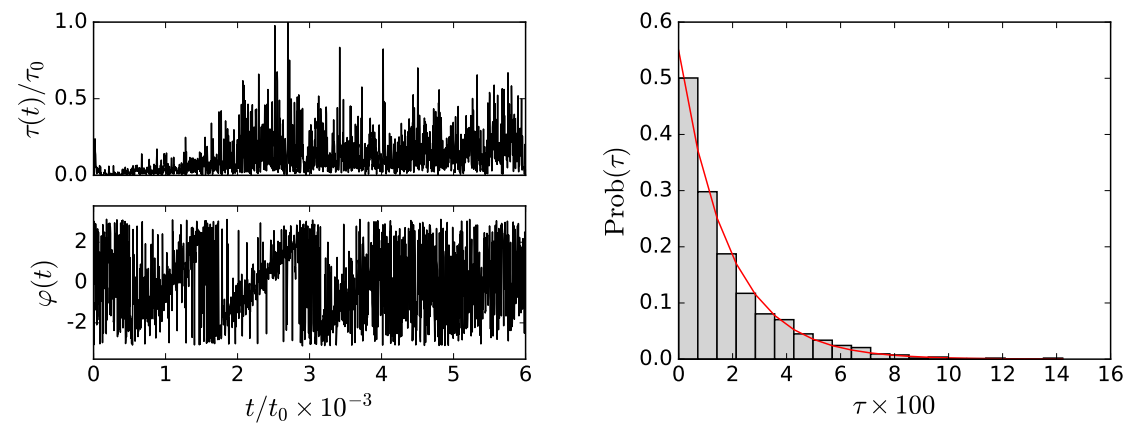

Fig. 5. Magnitude $\tau(t)$ and polar angle $\varphi(t)$ of the electron torque $\boldsymbol{\tau}\left(\boldsymbol{x}_{0}, t\right)=\boldsymbol{s}\left(\boldsymbol{x}_{0}, t\right) \times$ $\boldsymbol{S}\left(\boldsymbol{x}_{0}, t\right)$, computed at the skyrmion center. The magnitude probability distribution (left) compares with a Poissonian distribution, demonstrating the stochastic character of the electron torque term. ${ }^{10}$

at time $t$.

Equations (10)-(15) with (8) define the total Hamiltonien

$$
H=H_{S}+H_{e}+H_{\mathrm{sd}}
$$

of the coupled system of fixed and itinerant spins. To complete the model we must specify the dissipation effects. As in the micromagnetic model we consider the Gilbert relaxation coefficient $\alpha$. Other source of relaxation is related to the appearance of strong magnetization gradients in vortex and skyrmion cores regions, accompanying their switching. One simple derivation of the appropriated dissipation term $\boldsymbol{d}$ due to the exchange relaxation is through the Rayleigh dissipative function,

$$
\hbar \dot{H}_{S}=-\hbar \sum_{i} \boldsymbol{f}_{i} \cdot \dot{\boldsymbol{S}}_{i}=-\frac{1}{2} \sum_{i} \boldsymbol{f}_{i} \cdot \boldsymbol{d}=-\frac{\beta_{0}}{2} \sum_{i} \boldsymbol{f}_{i} \cdot \nabla^{2} \boldsymbol{f}_{i}
$$

where in the last equation we expanded $\boldsymbol{d}$ in powers of the gradients:

$$
\boldsymbol{d}_{i} \approx \alpha \boldsymbol{S}_{i} \times \boldsymbol{S}_{i} \times \boldsymbol{f}_{i}+\beta_{0} \nabla^{2} f_{i},
$$

and neglected the first term, proportional to the $\alpha$ term, because it is already present through the Gilbert relaxation. The second term, we keep, is the simplest vector one can form with the derivatives of $\boldsymbol{f}$, with one scalar proportionality constant $\beta_{0}$. Taking the exchange contribution to the dissipation vector $\boldsymbol{d}$, we obtain,

$$
\boldsymbol{d}_{i}=-\beta \nabla^{4} \boldsymbol{S}_{i}
$$

after a redefinition of the phenomenological coefficient, $\beta$. 
In summary, the dynamics of the fixed and itinerant spins is governed by the coupled equations,

$$
\begin{aligned}
\mathrm{i} \hbar \frac{\partial}{\partial t} \psi & =\left(H_{e}+J_{s} \boldsymbol{S}_{i} \cdot \boldsymbol{\sigma}\right) \psi \\
\hbar \frac{\partial}{\partial t} \boldsymbol{S}_{i} & =\boldsymbol{S}_{i} \times\left(\boldsymbol{f}_{i}-\alpha \boldsymbol{S}_{i} \times \boldsymbol{f}_{i}+J_{s}\left\langle\psi\left|\hat{\boldsymbol{s}}_{i}\right| \psi\right\rangle\right)-\beta \nabla^{4} S_{i},
\end{aligned}
$$

where

$$
\boldsymbol{f}_{i}=-\frac{\delta H_{S}}{\delta \boldsymbol{S}_{i}},
$$

we call Schrödinger-Landau-Lifshitz or SLL in short (here $\boldsymbol{f}_{i}$ has energy units). These equations no longer conserve the charge. In particular, the $\beta$ diffusion term tends to decrease the magnitude of the magnetization. However, even in the absence of the $\beta$ term, when the size of magnetization gradients becomes of the lattice step size the continuous description breaks down. Nevertheless, discretization effects are not enough to describe singularities in the magnetization field, coarse graining the microscopic lattice scales results in a continuous dynamics that conserves the topology of the initial texture. More importantly, the dynamics defined by is not strictly continuos in time, because to couple the two equations one must compute the electron spin mean value

$$
\left.\boldsymbol{s}\left(\boldsymbol{x}_{i}, t\right)=\left\langle\psi\left|\hat{\boldsymbol{s}}_{i}\right| \psi\right\rangle\right) .
$$

This quantum averaging (measure of the electron spin) involves information loss on the whole system, making the description nonlocal in space, and markovian in time (only the state at $t$ is used to compute the electron spin at $t+\Delta t$ ). In Fig. 5 we plot the time evolution of the electron torque and show that it follows effectively a Poissonian distribution. It was obtained by direct numerical integration of the SLL equations, for an initial skyrmion state that disappears at time around $t=5930$, as we study later in the section on the skyrmion collapse.

\section{Phenomenology of the topological transitions}

Using a numerical integration of the SLL equations, one can follow the evolution of the magnetization field and monitor the changes in the topological charge for various initial textures. We use the following conventions: The unit of length is as usual the lattice constant, which is here an effective tightbinding constant whose value can be tunned to fit the experimental properties of each material. It is typically below the nanometer. The hopping energy is of the order of the s-d coupling constant, and can be taken as the unit 


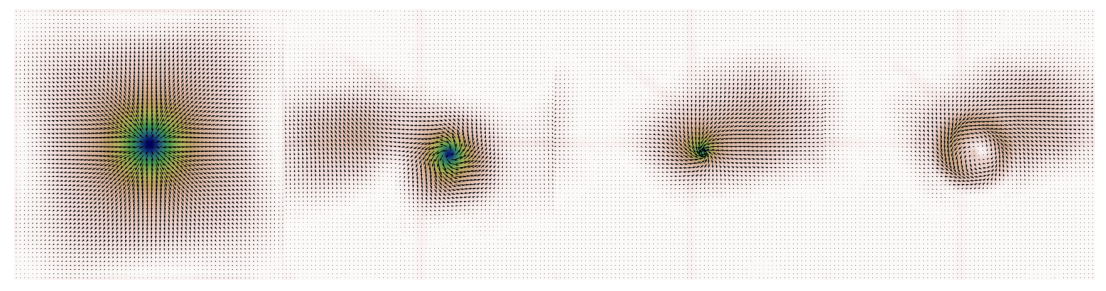

Fig. 6. Sequence showing the evolution of a skyrmion under a spin-polarized current, polarized in the opposite direction of its core. The initial skyrmion state of charge $Q=-1$, change into a state of trivial topology $Q=0$. The last frame show the expanding spin wave, generated at the transition time, in the ferromagnetic state. Times, 0, 4000, 5900 , and 6000 .

of energy. Typical values are $a \sim 0.3 \mathrm{~nm}$ and $\epsilon \sim 1 \mathrm{eV}$ for a ferromagnet, or $a \sim 0.5 \mathrm{~nm}$ and $\epsilon \sim 0.1 \mathrm{eV}$ for $\mathrm{MnSi}$, leading to a time scale of the order of $t_{0} \sim 1-10 \mathrm{fs}$, depending on the energy scale; based on these magnitudes the electric field is measured in units of $E_{0} \sim \epsilon /(e a) \approx 0.1-110^{9} \mathrm{~V} \mathrm{~m}^{-1}$ and the current in units of $I_{0} \sim e \epsilon / \hbar \approx 10-100 \mu \mathrm{A}$. In the numerical simulation we use $n_{e} \approx 0.1$ electrons per site, $E / E_{0} \approx 10^{-5}-510^{-4}$, and a spin polarization $B_{p}=0.1 \epsilon$; the coupling energies are $J_{s}=1, J=0.1,0.4$, $K=0, \pm 0.01, D=0,0.01$, in units of $\epsilon$, and the relaxation constant $\alpha=0.1$ (in all simulations).

In Fig. 6, we present a single skyrmion evolving under the action of a spin-up polarized current with weak exchange dissipation $(\beta=0.001)$, and a skyrmion lattice in Fig. 7, with a plane polarized current in a dissipationless case $(\beta=0)$. Both configurations evolve towards a final ferromagnetic state. In the case of the skyrmion lattice local cores having the topology of a vortex-antivortex pair, annihilate. The concomitant evolution of the topological charge is shown in Fig. 8. The collapse of the skyrmion core destroy its topological charge $Q=-1$, leaving a state of uniform magnetization whose charge is zero. The ferromagnetic transition of the skyrmion lattice proceeds by successive annihilation of vortex-antivortex pairs, which decrease the total charge by 1: each vortex having a charge of one half.

The skyrmion-ferromagnetic transition proceeds in several steps:

- After an initial transient, the current induces a skyrmion precession (periodic tilting of the core), and a displacement of the core (second frame in Fig. 6). The electron current density is highly inhomogeneous in the skyrmion core region, where itinerant spins are scattered off by the magnetization gradients. Indeed, electron 


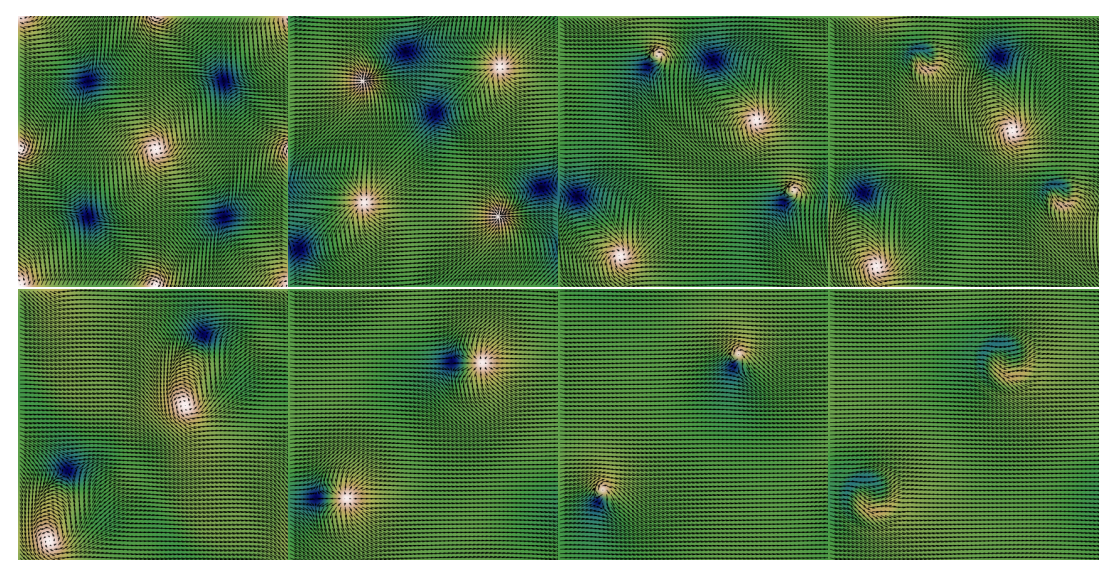

Fig. 7. Sequence of the skyrmion lattice evolution towards a ferromagnetic state, showing successive annihilations vortex-antivortex cores. The dynamics is driven by an inplane polarized electron current. The initial topological charge is $Q=4$, after annihilation of the first pair (last two frames of the first row), $Q=2$; the last frame is taken just after the annihilation of the second pair, $Q=0$, is already in the ferromagnetic phase. Times: $0,3060,4400,4756,5032,7032,9008$, and 9032. The initial frame was translated along the diagonal to center the skyrmion, initially situated at the upper left corner.

spins initially follow the fixed spins without penetrating into the central core; at the transition they build up a vortex-like structure, mostly in the plane, driving the switching of the core spins.

- Under the action of the polarized current the skyrmion moves following a trajectory that is mostly in the direction of the current, but also having a component perpendicular to its motion, associated with a gyrotropic force proportional to its topological charge. ${ }^{37}$ An illustration of this motion is shown in Fig. 9, where the trajectories of two skyrmions, one in the weak (exchange) dissipation regime (the same of Fig. 6), and the other in the exchange dissipationless regime $(\beta=0)$.

- The transition is characterized by the shrinking of the skyrmion core up to its complete collapse, which is followed by a burst of spin waves.

We see that, in both cases, the isolated skyrmion or the lattice, electron current inhomogeneities play an important role in the interaction of vortices. The usual approach of the spin-transfer torque completely neglects these effects. In particular the motion of the skyrmion can be excited by the in-plane current as in the lattice case, where the interaction between 

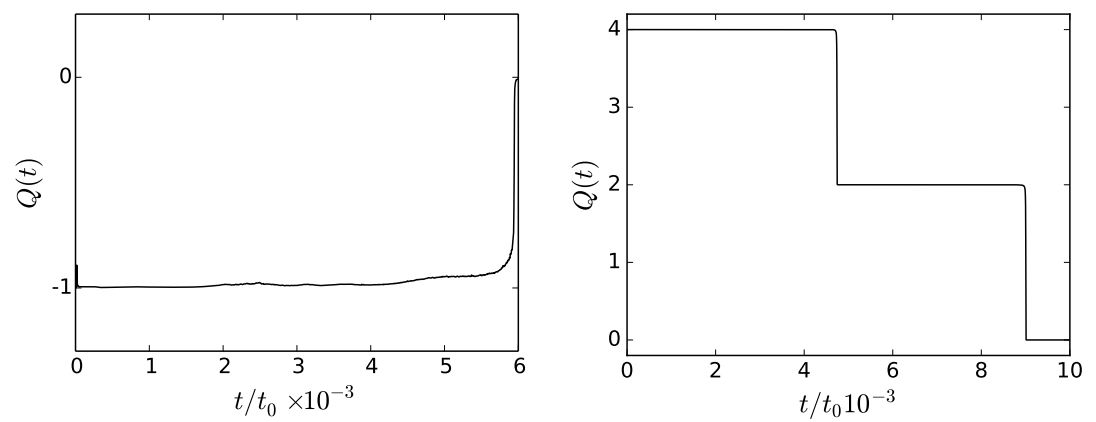

Fig. 8. Evolution of the charge and magnetization for an isolated skyrmion (left) and a skyrmion lattice (right).

the vortex cores favorises their motion, or, as can be seen in Fig. 9, by a $z$-polarized current. These electron current inhomogeneities are also related to spin field fluctuations that tend to organize into large scale structures around the cores. These relatively coherent electron spin structures play a role in the motion and internal degrees of freedom (spin waves) of the skyrmions. Recent experiments using a magnetic pulse to excite the skyrmion internal degrees of freedom in the $\mathrm{GHz}$ frequency range, show a subsequent gyrotropic motion of the cycloid type we observed in our simulations. ${ }^{38}$

More interestingly, the observation of the dynamics of the itinerant spins, suggests that the topological change might be related to the formation of a coherent structure of such spins, located around the skyrmion core, capable to reverse the central fixed spins by angular momentum transfer.

To investigate this mechanism we introduce a quantity we call the electron topological $b$-field, the itinerant spin equivalent of the topological charge density of the magnetization field:

$$
b=\boldsymbol{n} \cdot \partial_{x} \boldsymbol{n} \times \partial_{y} \boldsymbol{n}, \quad \boldsymbol{n}=\boldsymbol{s} /|\boldsymbol{s}| .
$$

which represents an effective magnetic field created by the electrons, projected in the direction of their spin. Precession of the fixed spins around this effective field, can induce their reversal. The idea is that the ferromagnetic transition involves the nucleation of an electron spin vortex with topological charge one $Q=1$ :

$$
Q(\text { ferro })=-1(\text { sky })+1(\text { elec })=0
$$

such that combined with the skyrmion minus one charge $Q=-1$, leads to 

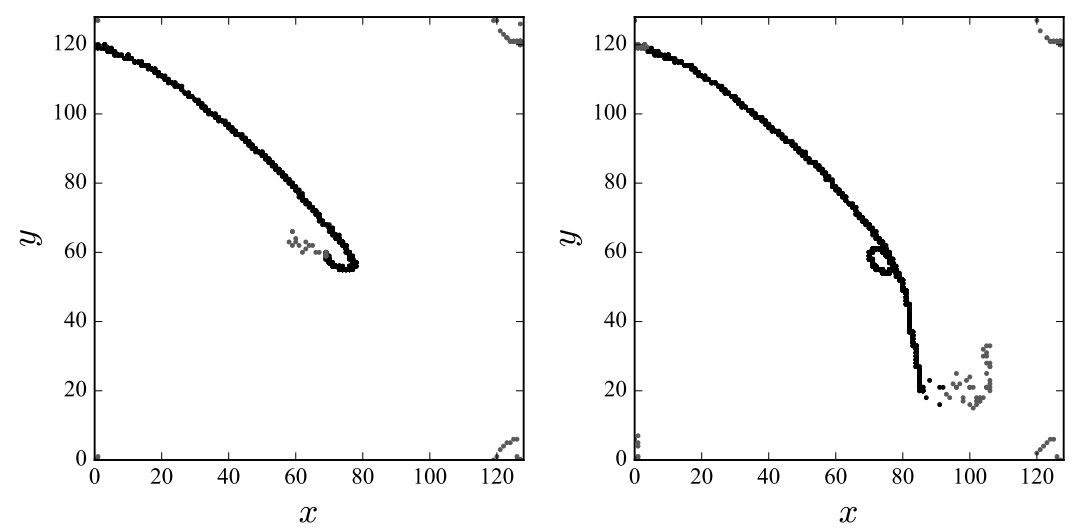

Fig. 9. Skyrmion trajectory in the presence of a polarized current; (left) $\beta=0.001$, (right) $\beta=0$. Points in gray correspond to the initial transient and after the topological transition, $t \approx 5930$ for the exchange dissipative case (left), and $t \approx 9820$ for the exchange dissipationless case (right).

the trivial state $Q=0$.

The picture represents in color the $b$-field together with the arrows of the itinerant spins; contours of the $z$-component of the fixed spin are also shown to locate the skyrmion core. The skyrmion core is reversed around time 5928, precisely at the moment where the electron spin vortex reach a maximum. Immediately later it disappears, leaving behind a strong spin wave visible also in the magnetization field. This observation tends to confirm the scenario of a topological transition triggered by the action of the electron current.

Even if at the topological transition the electron-spin field appears as organized in a large scale vortex, one should not forget that its dynamics is essentially stochastic. In particular, the electron-spin torque which drives the magnetization dynamics, obeys a Poissonian distribution, as confirmed numerically (c.f. Fig. 5). This fast dynamics translates into the slow and continuous dynamics of the fixed spins, by the formation of a finite time singularity, resolved by the discretization inherent to the lattice at the microscopic scales, and regularized by the exchange dissipation in the continuum limit. In the next section we investigate the dynamics of the topological transition. 


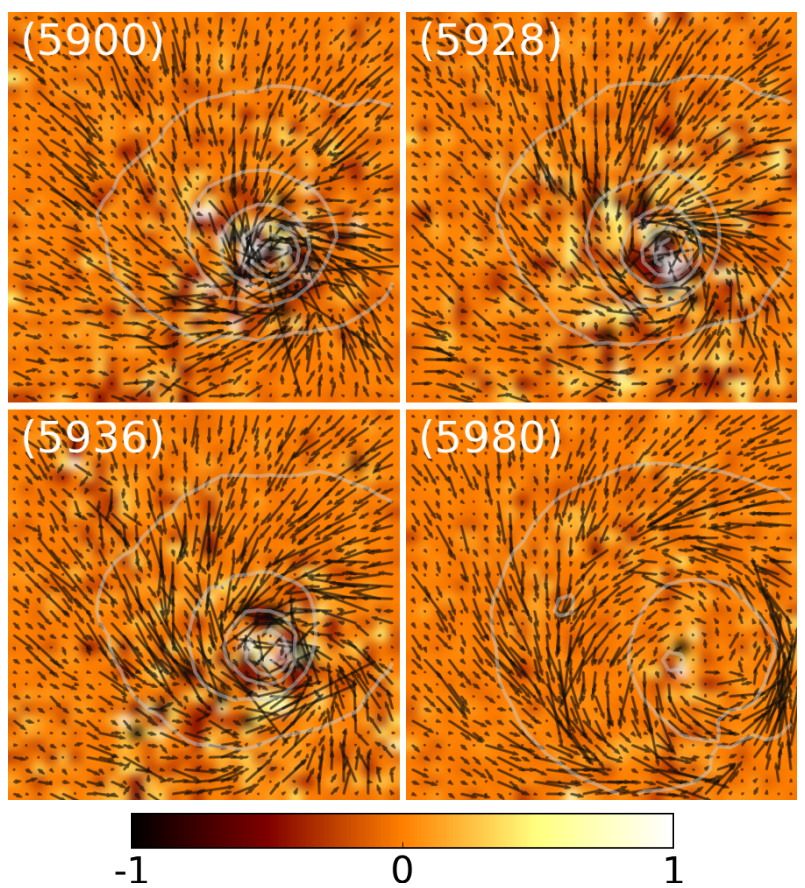

Fig. 10. Phenomenology of the topological transition. The topological b-field is represented in colors using the bottom colormap; arrows are the electron spins and contour lines the $z$ component of the magnetization (it shows the skyrmion core). Frame 5900 is just before the transition, 5928-5936 are around the transition, and 5980 is just after the transition. We observe that the electron spins are mostly oriented in the plane, and that at the transition, a strong electron topological charge is concentrated at the skyrmion core.

\section{Self-similar skyrmion collapse}

We focus now on the particular case of the transition between the skyrmion state and the ferromagnetic state, which involves the reversal of the skyrmion core magnetization, a process in which the variation of the topological charge is of 1 unit.

As we discussed in the previous section, the microscopic topological change can be related to a finite time singularity of the macroscopic magnetization field. We want to relate the topological change to the dynamics of the magnetization. We propose a continuous field magnetization effective model based on the self-similar vanishing of the skyrmion core characteristic length in a finite time. This singularity is stopped at the lattice scale, but appears as a genuine singularity in the continuum limit. Within this 
scenario we can neglect almost all terms in the Landau-Lifshitz equation, because at a collapsing skyrmion core only the external driving, here the electron current (spin-transfer torque), and the exchange interaction are dominant.

In order to obtain the relevant form of the Landau-Lifshitz equation we

- Assume that near the singularity the faster time is related to $\boldsymbol{S}$.

- Assume a self-similar evolution of the magnetization.

- Retain exchange and electron spin torque.

- Neglect dissipation, anisotropy and chirality.

The first assumption means that, in the continuum limit there is no characteristic time (or length) related to the topological transition: it appears as a discontinuity of the magnetization dynamics (as can be appreciated in the behavior of the topological charge, c.f. Fig. 8, right panel). Dissipation effects appear to be important at intermediate scales, but they seems to be not effective during the fast switching of the skyrmion core, at least for the parameters used in our simulations. Indeed, even for a large value of $\beta$, which affects the quantization of the topological charge, the size of the core abruptly goes to zero.

Taking the exchange interaction and the spin-transfer torque as the main mechanisms responsible for the singularity formation, the effective Landau-Lifshitz equation is in the continuous limit $\boldsymbol{S}_{i} \rightarrow \boldsymbol{S}(\boldsymbol{x}, t), S=1$,

$$
\frac{\partial}{\partial t} \boldsymbol{S}(\boldsymbol{x}, t)=\boldsymbol{S}(\boldsymbol{x}, t) \times\left[\boldsymbol{f}(\boldsymbol{x}, t)+\frac{J_{s}}{\hbar} \boldsymbol{s}(\boldsymbol{x}, t)\right]
$$

where the effective field,

$$
\boldsymbol{f}(\boldsymbol{x}, t)=\frac{J a^{2}}{\hbar} \nabla^{2} \boldsymbol{S}(\boldsymbol{x}, t),
$$

reduces to the exchange term. In the remaining of this section we mesure the spin in units of $\hbar$, and the energy in units of $J_{s}$ (to put physical units back one replaces $J \rightarrow J / J_{s}$, and add the necessary $\hbar$ constants). Under the assumptions specified above the last term, coupling with the current, appears essentially as an external magnetic field whose value will be adjusted to fit with the phenomenology of the topological transition, as described in Fig. 10.

To get some insight into the analytical properties of the magnetization dynamics it is convenient to transform the Landau-Lifshitz equation (22), using the stereographic projection. 


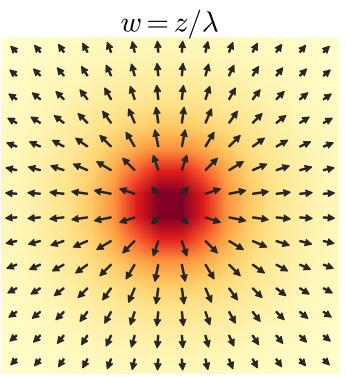

$w=\mathrm{e}^{\mathrm{i} \pi / 4} z / \lambda$

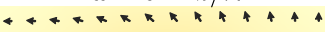

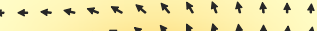

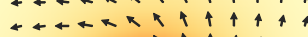

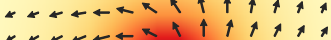

$x<x<x$

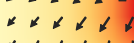

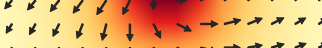

$\forall t+1+1 \rightarrow \rightarrow \rightarrow \rightarrow+\infty$

,

$\begin{array}{lll} & \end{array}$

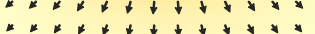

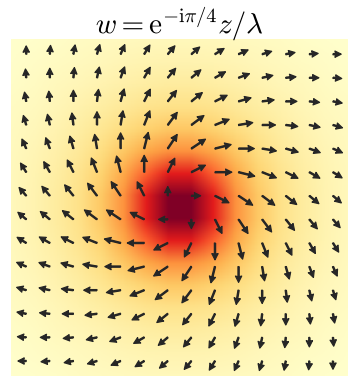

Fig. 11. Examples of skyrmions of charge $Q=1$, constructed from their stereographic projection $w(z) \rightarrow \boldsymbol{S}(\boldsymbol{x})$. As in the previous figures, arrows are for the vector plane components and colors are for the $S_{z}$ out of plane component (from yellow $S_{z}=-1$, to $\left.\operatorname{red} S_{z}=1\right)$.

The stereographic projection relates the vector field $S$ to the values of a complex function $w=w(z)$ through the transformation between the unit sphere and the complex plane $z, \boldsymbol{S}(\boldsymbol{x}, t) \rightarrow w(z, t)$, defined by,

$$
S_{x}=\frac{w+\bar{w}}{1+|w|^{2}}, S_{y}=\frac{1}{\mathrm{i}} \frac{w-\bar{w}}{1+|w|^{2}}, S_{z}=\frac{1-|w|^{2}}{1+|w|^{2}},
$$

and

$$
w=\frac{S_{x}+\mathrm{i} S_{y}}{1+S_{z}} .
$$

(the over bar denotes conjugation). In the stereographic plane the expression of a skyrmion reduces to a simple zero (or pole). In Fig. 11 we represent a skyrmion of charge $Q=1$ using simple formulas in the stereographic projection plane $z=x+\mathrm{i} y$ :

$$
w(z)=\mathrm{e}^{\mathrm{i} \varphi} \frac{z}{\lambda}
$$

where the parameter $\varphi$ controls the skyrmion winding and the parameter $\lambda$ controls the skyrmion core size. For a fixed $z$, the function $w \rightarrow \infty$ increases with a decreasing $\lambda \rightarrow 0$. We will use this fact to simplify the computation of the skyrmion geometry near the topological transition $\lambda \rightarrow 0$.

The stereographic projected Landau-Lifshitz equation becomes,

$$
\mathrm{i} \partial_{t} w=-J \partial \bar{\partial} w+\frac{2 J \bar{w}}{1+|w|^{2}} \partial w \bar{\partial} w-\frac{1}{2} s_{+}+s_{z} w+\frac{1}{2} s_{-} w^{2}
$$

where $s_{ \pm}=s_{x} \pm \mathrm{i} s_{y}$ and derivatives are taken over $z=x+\mathrm{i} y$. It is interesting to observe that this equation has the symmetry

$$
w \rightarrow 1 / w, s_{+} \leftrightarrow s_{-}, s_{z} \rightarrow-s_{z}
$$


that change the skyrmion charge $Q=1 \leftrightarrow-1$. Therefore, it is enough to consider the case $Q=1$ with $w \rightarrow z / \lambda$ for $\lambda \rightarrow 0$. In this case, near the singularity, the field $w$ is large and the relevant electron spin component is the one on the plane. The effective equation contains thus the in-plane component $s_{-}$of the electron spin (a term in $w^{2}$ ) and the exchange terms (proportional to $J$ )

$$
\mathrm{i} \partial_{t} w+J \nabla^{2} w=\frac{2 J}{w}(\nabla w)^{2}+\frac{1}{2} s_{-} w^{2}
$$

As mentioned above, the fast time for the change in the topology is related to the dynamics of the magnetization field $\boldsymbol{S}(\boldsymbol{x}, t)$; during this fast process one may consider that the electron field $\boldsymbol{s}(\boldsymbol{x}, t)$ is fixed. This assumption is in accordance with the behavior of the $b$-field; indeed, we observed (c.f. Fig. 10) that the electrons spin organizes in a coherent structure having the opposite charge of the skyrmion. It is precisely the precession of the magnetization around this effective field that trigger the charge change. Taking therefore, the $s_{-}$term as constant, equation (28) admits a selfsimilar solution,

$$
w(r, \varphi, t)=\frac{1}{\left(t_{*}-t\right)} f(X, \varphi), \quad X=\frac{r}{\left(t_{*}-t\right)^{1 / 2}},
$$

where $(r, \varphi)$ are polar coordinates $\left(z=r \mathrm{e}^{\mathrm{i} \varphi}\right)$, and $X$ is the self-similar variable. The singularity forms at a finite time $t \rightarrow t_{*}$, where $w$ diverges. The amplitude exponent equal to 1 , is fixed by the balance of the time derivative and the source term (in $s_{-}$), and the stretching length exponent $1 / 2$, results from the balance of the Laplacian with the time derivative. From these observation, one can estimate an order of magnitude of the singularity time, which in general will depend on the initial condition and is not therefore universal, as

$$
t_{*} \approx \frac{w(0)}{|\boldsymbol{s}(0)|} \sim \frac{\lambda(0)}{B_{p} n_{e} \sqrt{E}}
$$

where $\lambda$ is the skyrmion size, $B_{p} n_{e}$ is the density of the polarized electrons per site (unit length $a$ ) and $E$ the applied electric field (the velocity of electrons is $v \sim \sqrt{E})$. The stretching factor vanishes at the transition, which can be related to the vanishing of the core size $\lambda=\lambda(t) \sim\left(t_{*}-\right.$ $t)^{a}$, with $a$ an exponent to be determined, although a simple dimensional argument using $w \sim X /\left(t_{*}-t\right)$, gives $a=3 / 2$. Substitution of (29) into 
(28) gives,

$$
\begin{aligned}
\mathrm{i}\left(1+\frac{1}{2} X \partial_{X}\right) f=-J\left(\partial_{X X}\right. & \left.+\frac{1}{X} \partial_{X}+\frac{1}{X^{2}} \partial_{\varphi \varphi}\right) f \\
& +\frac{2 J}{f}\left[\left(\partial_{X} f\right)^{2}+\frac{1}{X^{2}}\left(\partial_{\varphi} f\right)^{2}\right]+\frac{s_{-}}{2} f^{2},
\end{aligned}
$$

where the condition $S_{z}(0,0)=1$ translates into the initial value $f(0)=0$. Contrary to $w, f$ is a smooth function near the origin, which means small $X$. Moreover, for large values of the argument we want $f$ diverges, which correspond to the uniform magnetization at infinity:

$$
\lim _{X \rightarrow 0} f(X, \phi)=0, \quad \lim _{X \rightarrow \infty} f(X, \phi)=\infty .
$$

Using the form

$$
f(X, \phi)=\mathrm{e}^{\mathrm{i} \varphi} F(X), \quad F(0)=0, \quad \lim _{X \rightarrow \infty} F(X)=\infty .
$$

we observe that the $\varphi$ dependence disappears from everywhere but the last term, which gives $F^{2} \mathrm{e}^{\mathrm{i} \varphi}$. Neglecting this small term (or equivalently, averaging over the angle), means that the transition process is universal, it do not depends explicitly on the driven force; the driving term enters through the specific value of the stretching exponent, and in the definition of $t_{*}$, but do not influences the form of the shape function $f$.

After these simplifications, we obtain an equation,

$$
\begin{aligned}
\left(1+\frac{1}{2} X \partial_{X}\right) F(X)+\frac{J}{X} \partial_{X} X \partial_{X} F(X)+ & J \frac{F(X)}{X^{2}} \\
& -\frac{2 J}{F(X)}\left[\partial_{X} F(X)\right]^{2}=0 .
\end{aligned}
$$

amenable at an analytic solution. ${ }^{10}$ Indeed, the simple substitution $g=1 / f$ leads to the linear equation:

$$
\mathrm{i}\left(1+\frac{x}{2} \partial_{X}\right) g(X)=\frac{J}{X} \partial_{X} X \partial_{X} g(X)
$$

which gives the general form, ${ }^{39}$

$$
\begin{aligned}
& g(X)=A G_{1,2}^{2,0}\left(\frac{\mathrm{i} r^{2}}{4 J\left|t_{*}-t\right|} \mid \begin{array}{c}
2 \\
-\frac{1}{2}, \frac{1}{2}
\end{array}\right) \\
& +B \mathrm{e}^{-\mathrm{i} X^{2} / 8 J} X^{3}\left[\left(1-\frac{6 \mathrm{i} J}{X^{2}}\right) J_{0}\left(\frac{X^{2}}{8 J}\right)+\mathrm{i}\left(1-\frac{2 \mathrm{i} J}{X^{2}}\right) J_{1}\left(\frac{X^{2}}{8 J}\right)\right]
\end{aligned}
$$

where $A, B$ are integration constants, $J_{0}$ and $J_{1}$ Bessel functions, and $G$ the Meijer function. ${ }^{40,41}$ From the boundary conditions of $F$, (31), one deduces 

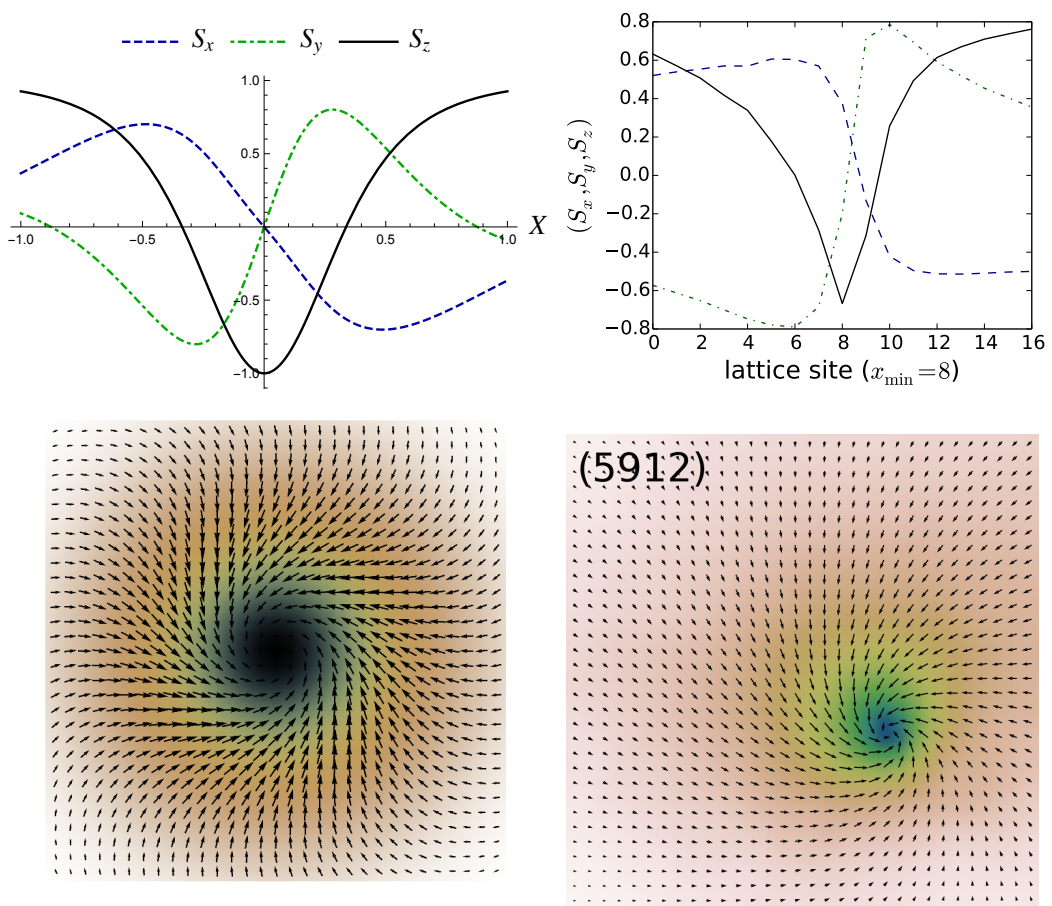

Fig. 12. Comparison of the analytical formula of the skyrmion self-similar shape (35) (left) and the numerical result of Fig. 6 (right). The top panel shows the components of the spin field near the skyrmion core, and the bottom panel the corresponding spin field.

that $B=0$, and only the Meijer function term subsists. Finally, collecting results the self-similar solution writes,

$$
w(r, \varphi, t)=\frac{C_{1} \mathrm{e}^{\mathrm{i} \varphi}}{\left|t_{*}-t\right|} G_{1,2}^{2,0}\left(\frac{\mathrm{i} r^{2}}{4 J\left|t_{*}-t\right|} \mid \begin{array}{c}
2 \\
-\frac{1}{2}, \frac{1}{2}
\end{array}\right)^{-1}
$$

where we came back to the original variables, and defined a new constant $C_{1}$ whose value must be determined from the fit to the external solution (far from the core, for example to match the inner solution to the correct external chirality). An asymptotic fit to (35) is given by a non-analytic complex function, representing a zero (like in the unperturbed skyrmion) whose length scale depends on time and its phase swirls rapidly with the distance to the center,

$$
w(r, \varphi, t) \approx \frac{C_{1}(1+\mathrm{i}) r \mathrm{e}^{\mathrm{i} \varphi}}{\left|t_{*}-t\right|^{3 / 2}} \mathrm{e}^{\mathrm{i} r^{2} / 4 J\left|t_{*}-t\right|},
$$


which allows an easy computation, using (24), of the skyrmion shape.

In Fig. 12 we compare the numerically computed skyrmion near the topological transition with formula (35). We use the function $1 / \bar{w}$ instead of $w$, to compare with a $Q=-1$ skyrmion, and choose a constant $C=J \mathrm{e}^{\mathrm{i} \varphi_{0}}$ (with $J=0.4$ and $\varphi_{0}=1.16$ ). The qualitative agreement is satisfying. It is worth noting that at variance with the regular spiraling around the skyrmion core of Fig. 11, the tilting of spins near the collapse time is more complex, involving in particular, lanes of almost parallel arrows converging to the periphery of the core; this is related to the radial dependency of the phase in the stereographic projection, absent in the simple pole (or zero) form of the skyrmion. Other important point is the law of the core shrinking scale:

$$
\lambda(t) \sim\left|t_{*}-t\right|^{3 / 2} .
$$

The exponent is different to the one obtained from the particular solution of (28),

$$
w(t)=\frac{2 \mathrm{i}}{s_{-}(z)}\left|t_{*}-t\right|^{-1},
$$

for which the exponent is 1 instead of $3 / 2$. However, this exact solution of (28), is interesting in itself and may also be relevant when the exchange interaction is overcame by the itinerant spin field (strong current limit). In such a case, the spatial distribution of the magnetization follows the spin current adiabatically and evolves rapidly in time with respect to the electrons (the opposite situation of the usual micromagnetic assumption of adiabatic electrons in the spin-transfer torque effect).

\section{Discussion and conclusions}

Magnetism is a beautiful phenomenon and a rich source of physical fundamental concepts, such as the concept of a field of forces first introduced by Faraday, with far reaching consequences in the elaboration of modern theories; it is also at the origin of important technological advances from motors to spintronics, and the cause of fascinating effects such the formation of vortices and skyrmion lattices in nanometric structures, the giant magnetoresistance or the spin-transfer torque.

In the present paper we covered a very restricted subject around micromagnetism and its generalization to account for topological transitions between different magnetization textures. We did not touch upon material 
properties or specific applications, and confined ourself to simple geometries and basic effective models. This approach allowed us to identify in the special case of the interaction of a spin polarized current with a skyrmion, the basic mechanisms governing the core switching and the change of the topological charge. We described a few experimental results, notably about the manipulation of skyrmions, showing that the spin-transfer torque effect is responsible of the skyrmion motion and eventually to its nucleation or annihilation.

More specifically, we proposed a model for the topological change based on a stochastic Landau-Lifshitz equation for the magnetization field, coupled with the Schrödinger equation for the electron current driven by an external electric field, and took into account, in addition to the usual Gilbert damping, an exchange dissipation effect. This model reveals the appearance of an electron vortex structure having the opposite topological charge of the skyrmion, at the moment of the core reversal.

From the analytical solution of the effective Landau-Lifshitz equation, valid near the transition, one may suggest a scenario for the topological change:

- An initial linear stage where the skyrmion shape remains close to its initial form with a time dependent phase describing its internal precession between different chirality states:

$$
w(z, t)=w_{0}(z) \mathrm{e}^{\mathrm{i} \varphi(z, t)}=\frac{z}{\lambda_{0}} \mathrm{e}^{-\mathrm{i} \int_{0}^{t} \mathrm{~d} t^{\prime} s_{z}\left(z, t^{\prime}\right)}
$$

where the electron $z$ spin component is considered independent of the magnetization dynamics. Under this condition, and assuming that the other spin components are zero $\left(s_{-}, s_{+}=0\right)$, this equation is an exact particular solution of the Landau-Lifshitz equation: any complex analytic function make the exchange terms vanish. During this stage the skyrmion wanders along a cycloid like trajectory, whose particular properties depend not only on the driven force, here the electron polarized current, but also on the dissipation mechanisms, here the exchange relaxation. The main influence of the exchange dissipation is on the life time of the skyrmion, and not on the path it follows.

- An intermediate stage, dominated by the electron spin in-plane components $\left(s_{x}, s_{y}\right)$, where a particular solution of the form (38)

$$
w(z, t)=\frac{2 \mathrm{i}}{s_{-}(z)}\left|t_{*}-t\right|^{-1}
$$


prevails: the magnetization spins precess around the effective field created by the itinerant spins, supposed to form a coherent structure in the vicinity of the skyrmion core. This intermediate stage is regularized by the exchange dissipation, which breaks the conservation of the topological charge through the variation of the magnetization magnitude.

- A final stage, dominated by the exchange interaction, where the skyrmion core collapses, characterized by the vanishing of the skyrmion core size at a rate faster than the quasi-adiabatic intermediate regime:

$$
w(z, t) \sim \frac{z}{\lambda(t)}, \quad \lambda(t) \sim\left|t_{*}-t\right|^{3 / 2}
$$

with an exponents of $3 / 2>1$. This stage is extremely fast, and seems to be immune to dissipation (Gilbert and exchange): it involves only microscopic scales and appears, in the continuum limit, as a finite time singularity.

It is important to note that in the absence of feedback between the electron current and the magnetization field, according to the exact solution (39) the skyrmion is stable and tends to follow adiabatically the out-ofplane component of the spin-polarized current. Only when an important in-plane component of the electron current develops, the skyrmion core can be perturbed and eventually reversed.

The microscopic model defined by equations (18)-(20) (SLL), prove to be able to describe the dynamics of vortices and skyrmions including the eventual changes in the magnetization field topology. The main physical ingredient of the model is the interaction between fixed and itinerant spins, allowing for inhomogeneities in the polarized current density that play a fundamental role in the topological transitions. The origin of these transition can be traced back to the lack of regularity of the dynamics, which is itself a consequence of the scale separation between the quantum effects relevant at the electron spin level, and the classical evolution of the fixed spins, whose continuum limit gives the usual Landau-Lifshitz micromagnetic equation.

Further work on three dimensional (Block point) singularities is in progress. 


\section{References}

1. L. D. Landau and E. M. Lifshitz, On the theory of the dispersion of magnetic permeability in ferromagnetic bodies, Phys. Zeitsch. der Sow. 8, 153 (1935), reprint: Ukr. J. Phys. 2008, Vol. 53, Special Issue, p.1422 .

2. A. A. Belavin and A. M. Polyakov, Metastable states of twodimensional isotropic ferromagnets, JETP Lett. 22, 245 (1975).

3. T. H. R. Skyrme, Particle states of a quantized meson field, Proceedings of the Royal Society of London A: Mathematical, Physical and Engineering Sciences 262, 237 (1961).

4. H.-B. Braun, Topological effects in nanomagnetism: from superparamagnetism to chiral quantum solitons, Advances in Physics 61, 1 (2012/06/04 2012).

5. S. Muhlbauer, B. Binz, F. Jonietz, C. Pfleiderer, A. Rosch, A. Neubauer, R. Georgii and P. Boni, Skyrmion Lattice in a Chiral Magnet, Science 323, 915 (2009).

6. X. Z. Yu, Y. Onose, N. Kanazawa, J. H. Park, J. H. Han, Y. Matsui, N. Nagaosa and Y. Tokura, Real-space observation of a twodimensional skyrmion crystal, Nature 465, 901 (06 2010).

7. T. Shinjo, T. Okuno, R. Hassdorf, K. Shigeto and T. Ono, Magnetic vortex core observation in circular dots of permalloy, Science $\mathbf{2 8 9}, 930$ (2000).

8. N. Romming, C. Hanneken, M. Menzel, J. E. Bickel, B. Wolter, K. von Bergmann, A. Kubetzka and R. Wiesendanger, Writing and deleting single magnetic skyrmions, Science 341, 636 (08 2013).

9. R. G. Elías and A. D. Verga, Topological changes of two-dimensional magnetic textures, Phys. Rev. B 89, p. 134405 (2014).

10. A. D. Verga, Skyrmion to ferromagnetic state transition: A description of the topological change as a finite-time singularity in the skyrmion dynamics, Phys. Rev. B 90, 174428 (2014).

11. R. P. Cowburn, D. K. Koltsov, A. O. Adeyeye, M. E. Welland and D. M. Tricker, Single-domain circular nanomagnets, Phys. Rev. Lett. 83, 1042 (1999).

12. A. N. Bogdanov and D. A. Yablonskii, Thermodynamically stable "vortices" in magnetically ordered crystals. The mixed state of magnets, Sov. Phys. JETP 68, 101 (1989), Russian original - ZhETF, Vol. 95, No. 1, p. 178, January 1989.

13. A. N. Bogdanov, U. K. R`ssler and C. Pfleiderer, Modulated and lo- 
calized structures in cubic helimagnets, Physica B: Condensed Matter 359-361, 1162 (2005).

14. A. Neubauer, C. Pfleiderer, B. Binz, A. Rosch, R. Ritz, P. G. Niklowitz and P. Böni, Topological hall effect in the $a$ phase of mnsi, Phys. Rev. Lett. 102, p. 186602 (May 2009).

15. M. Finazzi, M. Savoini, A. R. Khorsand, A. Tsukamoto, A. Itoh, L. Duò, A. Kirilyuk, T. Rasing and M. Ezawa, Laser-induced magnetic nanostructures with tunable topological properties, Phy. Rev. Lett. 110, 177205 (04 2013).

16. X. Z. Yu, N. Kanazawa, W. Z. Zhang, T. Nagai, T. Hara, K. Kimoto, Y. Matsui, Y. Onose and Y. Tokura, Skyrmion flow near room temperature in an ultralow current density, Nat Commun 3, p. 988 (08 2012).

17. Y. Zhou and M. Ezawa, A reversible conversion between a skyrmion and a domain-wall pair in a junction geometry, Nature Communications 5 (08 2014).

18. R. Tomasello, E. Martinez, R. Zivieri, L. Torres, M. Carpentieri and G. Finocchio, A strategy for the design of skyrmion racetrack memories, Scientific Reports 4, 6784 EP (10 2014).

19. Y. Tokunaga, X. Z. Yu, J. S. White, H. M. Ronnow, D. Morikawa, Y. Taguchi and Y. Tokura, A new class of chiral materials hosting magnetic skyrmions beyond room temperature, Nat Commun 6 (07 2015).

20. B. Van Waeyenberge, A. Puzic, H. Stoll, K. W. Chou, T. Tyliszczak, R. Hertel, M. Fahnle, H. Bruckl, K. Rott, G. Reiss, I. Neudecker, D. Weiss, C. H. Back and G. Schutz, Magnetic vortex core reversal by excitation with short bursts of an alternating field, Nature 444, 461 (11 2006).

21. R. Hertel, S. Gliga, M. Fahnle and C. M. Schneider, Ultrafast nanomagnetic toggle switching of vortex cores, 98, p. 117201 (Mar 2007).

22. R. Hertel and C. M. Schneider, Exchange explosions: Magnetization dynamics during vortex-antivortex annihilation, 97, p. 177202 (Oct 2006).

23. Q. F. Xiao, J. Rudge, E. Girgis, J. Kolthammer, B. C. Choi, Y. K. Hong and G. W. Donohoe, Dynamics of magnetic vortex core switching in fe nanodisks by applying in-plane magnetic field pulse, Journal of Applied Physics 102, 103904 (2007).

24. R. G. Elías and A. D. Verga, Magnetization structure of a bloch point singularity, Eur. Phys. J. B 82, 159 (2011). 
25. J. C. Slonczewski, Current-driven excitation of magnetic multilayers, Journal of Magnetism and Magnetic Materials 159, L1 (1996).

26. L. Berger, Emission of spin waves by a magnetic multilayer traversed by a current, Phys. Rev. B $\mathbf{5 4}$ (1996).

27. E. B. Myers, D. C. Ralph, J. A. Katine, R. N. Louie and R. A. Buhrman, Current-induced switching of domains in magnetic multilayer devices, Science 285, 867 (1999).

28. J. A. Katine, F. J. Albert, R. A. Buhrman, E. B. Myers and D. C. Ralph, Current-driven magnetization reversal and spin-wave excitations in co /cu /co pillars, Phys. Rev. Lett. 84, 3149 (Apr 2000).

29. L. Gan, S. Chung, K. Aschenbach, M. Dreyer and R. Gomez, Pulsedcurrent-induced domain wall propagation in permalloy patterns observed using magnetic force microscope, Magnetics, IEEE Transactions on 36, 3047 (Sep 2000).

30. Y. B. Bazaliy, B. A. Jones and S.-C. Zhang, Modification of the landaulifshitz equation in the presence of a spin-polarized current in colossaland giant-magnetoresistive materials, Phys. Rev. B 57, R3213 (1998).

31. N. Nagaosa and Y. Tokura, Topological properties and dynamics of magnetic skyrmions, Nature Nanotechnology 8, 899 (12 2013).

32. P. Bruno, V. K. Dugaev and M. Taillefumier, Topological Hall effect and Berry phase in magnetic nanostructures, Phys. Rev. Lett. 93, p. 096806 (2004).

33. J. Zang, M. Mostovoy, J. H. Han and N. Nagaosa, Dynamics of skyrmion crystals in metallic thin films, Phys. Rev. Lett. 107, p. 136804 (Sep 2011).

34. J. Miltat and A. Thiaville, Ferromagnetism: Vortex cores-smaller than small, Science 298, 555 (10 2002).

35. A. Thiaville, J. M. García, R. Dittrich, J. Miltat and T. Schrefl, Micromagnetic study of bloch-point-mediated vortex core reversal, Phys. Rev. B 67, p. 094410 (2003).

36. V. G. Baryakhtar, B. A. Ivanov, A. L. Sukstanskii and E. Y. Melikhov, Soliton relaxation in magnets, Phys. Rev. B 56, 619 (1997).

37. A. A. Thiele, Steady-state motion of magnetic domains, Phys. Rev. Lett. 30 (1973).

38. F. Buttner, C. Moutafis, M. Schneider, B. Kruger, C. M. Gunther, J. Geilhufe, C. v. K. Schmising, J. Mohanty, B. Pfau, S. Schaffert, A. Bisig, M. Foerster, T. Schulz, C. A. F. Vaz, J. H. Franken, H. J. M. Swagten, M. Klaui and S. Eisebitt, Dynamics and inertia of skyrmionic spin structures, Nat Phys 11, 225 (03 2015). 
39. Wolfram Research, Inc., Mathematica, Champaign, Illinois (2016), Version 10.3, www.wolfram.com.

40. R. Beals and J. Szmigielski, Meijer g-functions: A gentle introduction, Notices of the AMS 60, 866 (2013).

41. F. Olver, NIST Handbook of Mathematical Functions (Cambridge University Press, New York, NY, 2010), Online version: http://dlmf.nist.gov/. 